\title{
Retrospective analysis of dose delivery in intra-operative high dose rate brachytherapy
}

\author{
Moonseong Oh, Jaiteerth S. Avadhani, Harish K. Malhotra, Barbara Cunningham, \\ Patrick Tripp, Wainwright Jaggernauth, Matthew B. Podgorsak
}

Department of Radiation Medicine, Roswell Park Cancer Institute, Buffalo, New York, USA

Background. This study was performed to quantify the inaccuracy in clinical dose delivery due to the incomplete scatter conditions inherent in intra-operative high dose rate (IOHDR) brachytherapy.

Methods. Treatment plans of 10 patients previously treated in our facility, which had irregular shapes of treated areas, were used. Treatment geometries reflecting each clinical case were simulated using a phantom assembly with no added build-up on top of the applicator. The treatment planning geometry (full scatter surrounding the applicator) was subsequently simulated for each case by adding bolus on top of the applicator.

Results. For geometries representing the clinical IOHDR incomplete scatter environment, measured doses at the $5 \mathrm{~mm}$ and $10 \mathrm{~mm}$ prescription depths were lower than the corresponding prescribed doses by about $7.7 \%$ and $11.1 \%$, respectively. Also, for the two prescription methods, an analysis of the measured dose distributions and their corresponding treatment plans showed average decreases of $1.2 \mathrm{~mm}$ and $2.2 \mathrm{~mm}$ in depth of prescription dose, respectively.

Conclusions. Dosimetric calculations with the assumption of an infinite scatter environment around the applicator and target volume have shown to result in dose delivery errors that significantly decrease the prescription depth for IOHDR treatment.

Key words: intraoperative period; brachytherapy; radiotherapy dosage

\section{Introduction}

Intra-operative radiation therapy (IORT) is the delivery of a relatively high dose of radiation to the tumour bed or residual disease at the time of surgical resection. The benefit of this technique is the po-

Received 12 October 2007

Accepted 19 October 2007

Correspondence to: Matthew B. Podgorsak, Department of Radiation Medicine, Roswell Park Cancer Institute, Elm \& Carlton Streets, Buffalo, NY 14263, USA; Phone: +1 716845 1536; Fax: +1 716845 7616; E-mail: moonseong.oh@roswellpark.org tential to shield or displace normal tissues thus minimally exposing them to radiation. Clinically, IORT has been used as an adjuvant to surgery and/or fractionated external beam radiation therapy for locally advanced cancers of the abdomen, pelvis, head and neck, brain, thorax, and extremities. ${ }^{1-7}$ Historically, linear accelerators employing electron beams were used for IORT. ${ }^{5,}$ 8-10 However, there has been an interest in applying high dose rate (HDR) brachytherapy for this purpose. ${ }^{11-14}$

In intra-operative high dose rate (IOHDR) brachytherapy, applicators are 
Table 1. Foreshortening of the depth of prescription dose

\begin{tabular}{cccccc}
\hline Patients & $\begin{array}{c}\text { Intended } \\
\text { prescription } \\
\text { depth (mm) }\end{array}$ & $\begin{array}{c}\text { Prescription } \\
\text { dose (cGy) }\end{array}$ & $\begin{array}{c}\text { d (Prescription } \\
\text { distance from } \\
\text { the plan }(\mathbf{m m}))\end{array}$ & $\begin{array}{c}\mathbf{d}^{\prime} \text { (Actual } \\
\text { distance }(\mathbf{m m}))\end{array}$ & $\begin{array}{c}\mathbf{d}^{-d^{\prime}} \\
(\mathbf{m m})\end{array}$ \\
\hline A & 10 & 1500 & 9.8 & 7.8 & 2.0 \\
B & 10 & 1000 & 10 & 7.6 & 2.4 \\
C & 10 & 500 & 10 & 7.7 & 2.3 \\
D & 10 & 1000 & 9.9 & 8.0 & 1.9 \\
E & 10 & 1250 & 10 & 7.9 & 2.1 \\
F & 10 & 750 & 10 & 7.8 & 2.2 \\
G & 10 & 1250 & 10 & 7.8 & 2.2 \\
H & 10 & 750 & 10 & 7.7 & 2.3 \\
I & 5 & 1250 & 5 & 3.8 & 1.2 \\
J & 5 & 1500 & 5 & 3.7 & 1.3 \\
\hline
\end{tabular}

secured directly to the residual tumour or tumour bed. The region anterior to the applicators is mostly air with significantly less scattering properties than tissue. The dose computation algorithm in commercial treatment planning system assumes that the applicators are surrounded by an infinite scatter medium. ${ }^{15}$ This assumption, however, is strictly valid only in cases of interstitial and intracavitary brachytherapy and may lead to an over-estimation of the dose in the case of IOHDR brachytherapy.

In a recent publication, ${ }^{16}$ we have shown that this lack of scatter from one side of the applicator has the potential of leading to significant underdosage during treatments. Our measurements showed that underdosages at two planned prescription depths (5 $\mathrm{mm}$ and $10 \mathrm{~mm}$ ) were $8.5 \%$ and $12.5 \%$ for each of the conventional treatment geometries studied (applicators with surface areas of 4,7 , and $12 \mathrm{~cm}^{2}$ ). In a clinical environment, IOHDR brachytherapy treatments typically involve irregular surface areas and there has been concern whether the previous published results with standard irradiation geometries can be ported to these clinical situations as well. In the present study, we have used an experimental approach to quantify the magnitude of underdosage in clinical cases with irregularly shaped applicators.

\section{Materials and methods}

In this retrospective study, the treatment plans of 10 consecutive patients previously treated at our facility were analyzed. Eight patients had a prescription depth of $10 \mathrm{~mm}$ where the therapy was delivered out using applicators (Freiburg Flap Applicator, Freiburg, Germany) consisting of a contiguous array of $5 \mathrm{~mm}$ radius plastic spherical beads, which have a provision to insert multiple nylon catheters separated by 10 $\mathrm{mm}$. The remaining two patients were treated without an applicator, resulting in a prescription depth of $5 \mathrm{~mm}$. In our practice, the prescription depth is defined as the distance between the center of the source dwell positions and the treatment plane. The clinical set up of a representative IOHDR brachytherapy treatment is shown in Figure 1. In this study, the prescribed dose varied from 5 Gy to 15 Gy (Table 1). The treated areas were irregular in shape, covering surface areas of $8 \mathrm{~cm}^{2}$ to $180 \mathrm{~cm}^{2}$ 


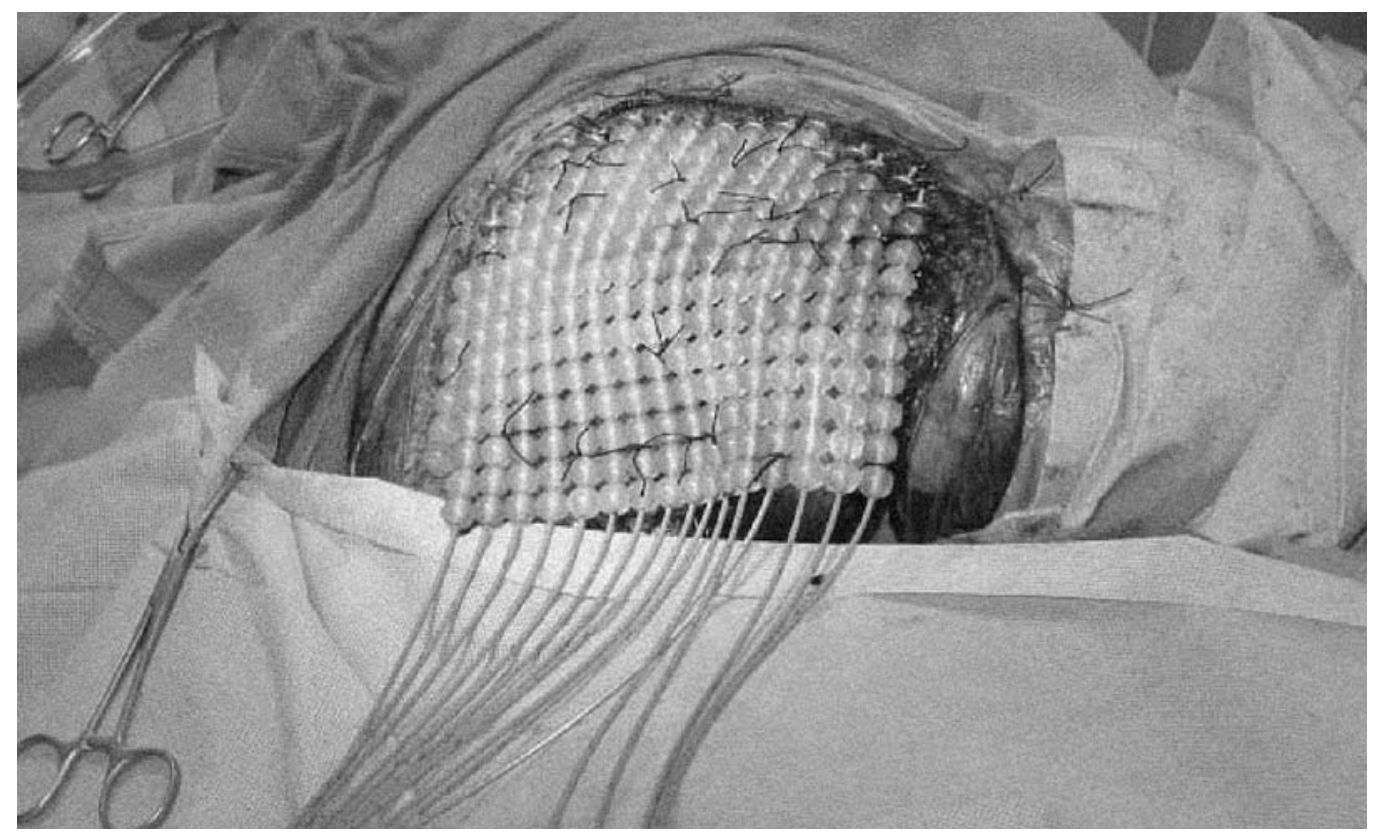

Figure 1. Clinical setup of a representative IOHDR case.

and consisted of 3 to 16 catheters, depending on the size of the target volumes.

The clinical treatment plans for each patient were restored to the planning system (Plato, v. 14.2, Nucletron, Columbia, MD)

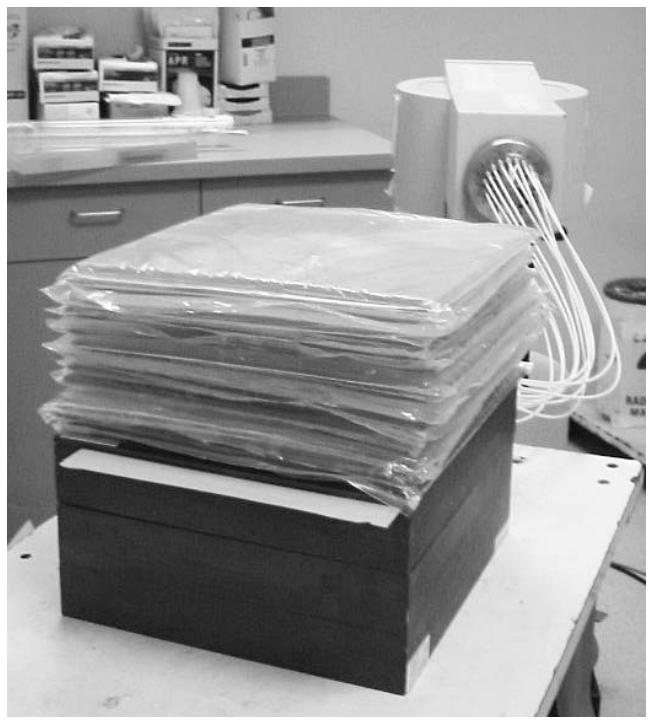

and were renormalized to deliver a dose of 200 cGy to the original prescription depth. The measurement setup is shown in Figure 2. For patients with a $10 \mathrm{~mm}$ prescription depth, the treatment delivery was simulated

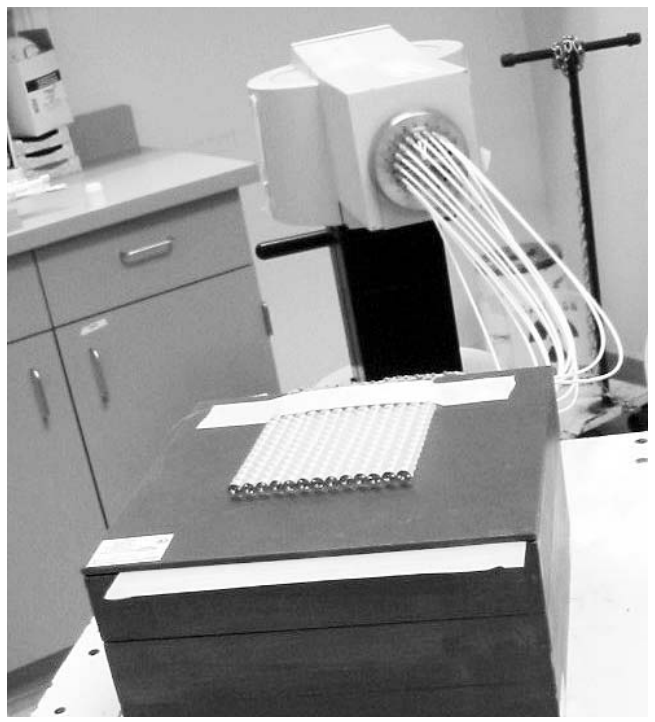

Figure 2. The measurement setup: (a) Full scatter environment, (b) No scatter environment.

Radiol Oncol 2007; 41(4): 188-95. 
by inserting $5 \mathrm{~mm}$ bolus material posterior to the applicator to achieve the prescribed distance from the center of the source to a piece of radiographic film. EDR2 films used to get dose profiles were placed on top of a solid water phantom $(30 \mathrm{~cm} \times 30 \mathrm{~cm} \times$ $15 \mathrm{~cm}$ ) and full scatter conditions were obtained by putting $15 \mathrm{~cm}$ of bolus material on the top of the applicator.

The H \& D curve for EDR2 film for Ir192 was generated using a reference applicator having a treatment area of $7 \times 7 \mathrm{~cm}^{2}$ and a prescription depth of $10 \mathrm{~mm}$ with the prescribed dose varying from 0 to $400 \mathrm{cGy}$ (Figure 3). Fifteen centimeters bolus on top of the applicator was used to simulate the

\section{H \& D Curve}

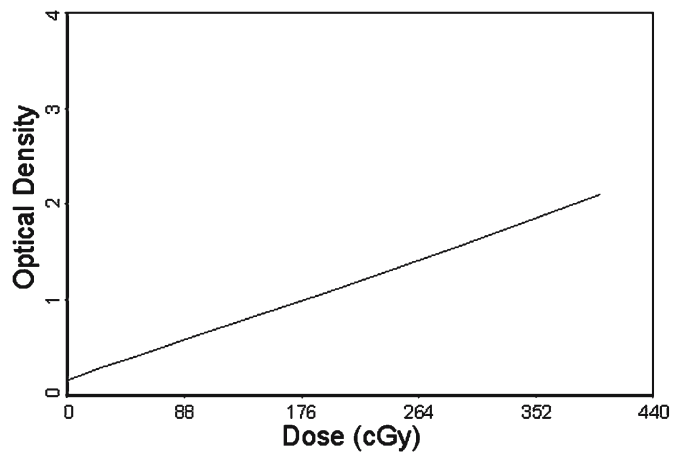

Figure 3. EDR2 H\&D dose calibration curve for dose up to $400 \mathrm{cGy}$.

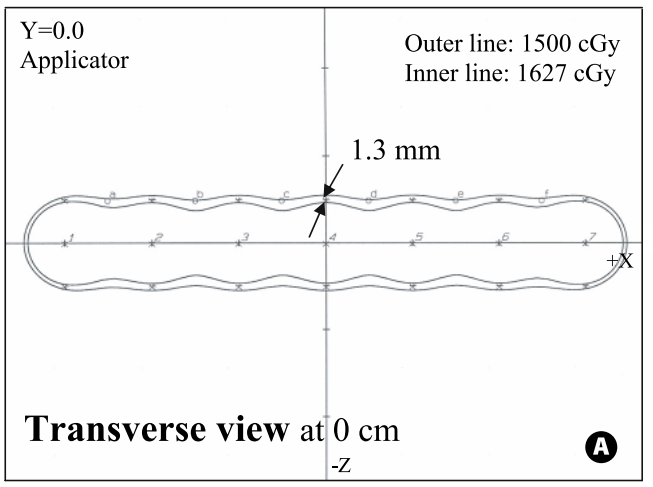

full scattering environment. ${ }^{16} \mathrm{H}$ \& D curve films as well as the measurement films were processed in quick succession to reduce processor dependent uncertainties. A Kodak RP X-OMAT film processor (Model M6B) was used for developing all films. These films were then scanned using a Vidar VXR-16 Dosimetry PRO ${ }^{\mathrm{TM}}$ film scanner (Vidar Systems Corporation, VA) and analyzed using the RIT 113 film Dosimetry system (version 3.14).

\section{Results}

Figure 4 shows the optimized dose distributions in transverse planes for 2 representative patients having a prescription dose of 15 \& 7.5 Gy delivered to the prescription depths of 5 and $10 \mathrm{~mm}$, respectively. In the case of incomplete scatter, the prescription dose was actually delivered to a point that was shorter than the intended prescription depth. The magnitude of this shift was found to be a function of the prescription depth and was about $1.3 \mathrm{~mm}$ and $2.2 \mathrm{~mm}$ for the prescription depths of $5 \mathrm{~mm}$ and $10 \mathrm{~mm}$, respectively, as shown in Table 1 . Also, Table 1 shows the average distance differences between the measured and planned prescription dose lines. As can be

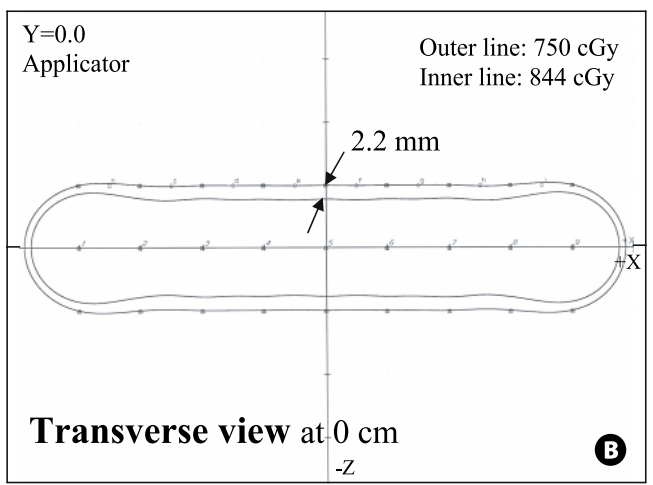

Figure 4. Optimized dose distribution in an axial plane (outer line: prescribed dose line, inner line: actual depth of prescription dose): (a) For the plan with a $5 \mathrm{~mm}$ prescription depth of patient $\mathrm{J}$, (b) for the plan with a $10 \mathrm{~mm}$ prescription depth of patient $\mathrm{F}$. 
Table 2. Measured doses in clinical (no added scatter) and treatment planning (full scatter) geometries

\begin{tabular}{cccccc}
\hline \multirow{2}{*}{ Patients } & $\begin{array}{c}\text { Prescription } \\
\text { depth }(\mathbf{m m})\end{array}$ & $\begin{array}{c}\text { Intended Dose } \\
(\mathbf{c G y})\end{array}$ & \multicolumn{2}{c}{ Delivered Dose (cGy) } & Underdosage (\%) \\
\hline A & 10 & 200 & 174.15 & 194.47 & 10.45 \\
B & 10 & 200 & 178.21 & 198.31 & 10.14 \\
C & 10 & 200 & 180.99 & 205.6 & 11.97 \\
D & 10 & 200 & 173.79 & 194.06 & 10.45 \\
E & 10 & 200 & 174.48 & 197.49 & 11.65 \\
F & 10 & 200 & 172.92 & 197.44 & 12.42 \\
G & 10 & 200 & 175.13 & 196.32 & 10.79 \\
H & 10 & 200 & 172.91 & 194.51 & 11.10 \\
\hline & Average dose for 8 patients & & $\mathbf{1 7 5 . 3 2}$ & $\mathbf{1 9 7 . 2 8}$ & $\mathbf{1 1 . 1 3}$ \\
\hline I & 5 & 200 & 184.15 & 199.32 & 7.61 \\
J & 5 & 200 & 181.91 & 197.07 & 7.69 \\
\hline
\end{tabular}

seen from Table 1, the distance between the planned and the measured isodose line is a function of the prescription depth.

As expected, the actual doses delivered to the prescription points were $10.5 \%$ to $12.4 \%$ (average 11.1\%) lower than prescribed doses for the cases with the prescription depth of $10 \mathrm{~mm}$ and were about $7.7 \%$ lower with the prescription depth of $5 \mathrm{~mm}$ (Table 2 and Figure 5). The magnitude of this under-

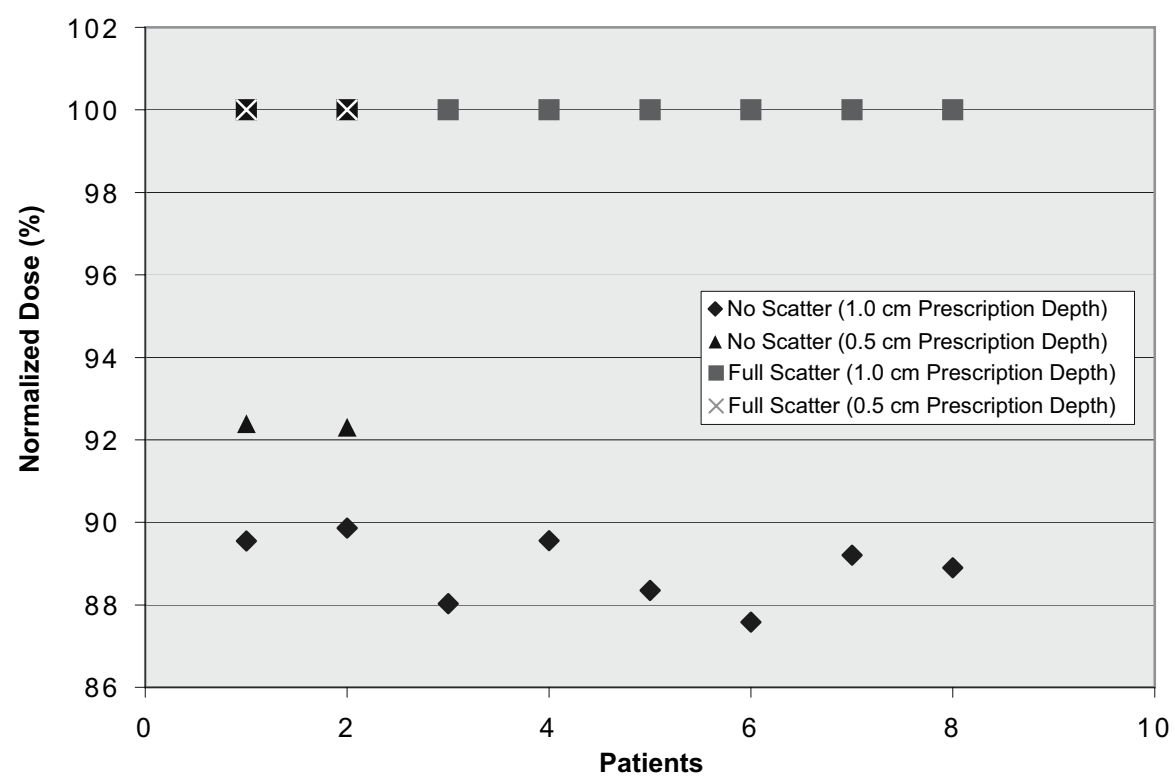

Figure 5. Comparison of measured doses for both $10 \mathrm{~mm}$ and $5 \mathrm{~mm}$ prescription depths; the doses are normalized to the corresponding doses with full scattering. 
dosage was dependent on the prescription depth and independent of the treatment area. In our earlier paper, ${ }^{16}$ we had reported on this underdosage for square applicator geometries $\left(4 \times 4,7 \times 7 \& 12 \times 12 \mathrm{~cm}^{2}\right)$. Our present work, using the actual patient plans with irregular geometry of applicators/source configurations, has also given similar results, thereby, further strengthening our earlier observation that the underdosage is a function of prescription depth and does not depend on either the shape or the size of the treatment area. This underdosage was also uniformly spread throughout the treatment area as can be seen from Figure 6 where delivered dose profiles with and without backscatter materials are plotted for a representative patient. For this particular patient, the prescription was 10 $\mathrm{mm}$.

As shown in Table 2, measured doses with the full scattering were very close to the intended dose of 200 cGy, with averages of 197.3 cGy and 198.2 cGy for $10 \mathrm{~mm}$ and $5 \mathrm{~mm}$ prescription depths, respectively. Incidentally the measured dose values are within the $\pm 3 \%$ uncertainty expected from film dosimetry ${ }^{17}, 18$ when carried out in a controlled environment.

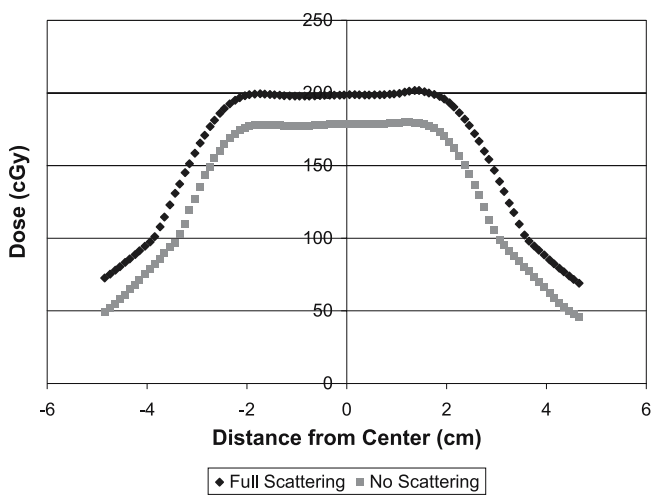

Figure 6. Delivered dose profiles with and without scatter in $10 \mathrm{~mm}$ prescription depth for patient $\mathrm{B}$.

\section{Discussion}

In case of IOHDR treatments which usually have a single plane and no crossing at the end of catheters, this results in plans in which the dwell positions at the periphery get higher dwell times than at the center. Thus, this type of inhomogeneous dwell time distribution results in optimized treatment plans which give more homogeneous dose throughout the desired target area. In case of IOHDR brachytherapy treatments, this target is an area encompassed by the prescription point. In arriving at the optimized plan, the planning system assumes that the applicators are surrounded by infinite scatter material. However, this assumption becomes invalid when one side of the applicator is exposed only to air, as is frequently the case in IOHDR brachytherapy. Therefore, a significant underdosage due to the lack of scatter would potentially be delivered to the target volume.

In most IOHDR treatments, typical geometry does not include full scatter on the opposite side of the applicator from the treatment area. This results in an over-estimation of the delivered dose in the target volume by the treatment planning system due to the over-simplistic assumption of the full scatter during the dose computation. One can think of correcting for this underdosage by augmenting the scatter environment by placing a scattering medium on the top of the applicators during IOHDR procedure. However, the addition of bolus material during the IORT is not always feasible. A real danger is the weight of the added bolus compressing critical structures in and around the target area. A simple approach will be to account for this underdosage during the treatment planning stage by either prescribing to an appropriate isodose level or by shifting the prescription points deeper into the target. 
We have demonstrated that without the typical scatter environment, actual dose delivered can be different from prescription dose. But how this "underdosing" would potentially change clinical outcome is less clear. IOHDR clinical experience has been built on long-established techniques that do not account for the suboptimal scatter environment. While our measurements suggest that typical HDR treatment planning systems underestimate the actual dose delivered by $7.7 \%$ at $5 \mathrm{~mm}$ and $11.1 \%$ at 10 $\mathrm{mm}$, we do not know whether this "underdosage" would change clinical outcome. Our study has demonstrated that the historical data available using electron IORT cannot be compared with IOHDR without factoring this underdosage. Peripheral nerve and other normal structure IOHDR recommended tolerance doses have been established by clinical experience. For target prescription dose and for normal structure dose tolerance, we hope that our proposed more accurate dosimetry would be clinically meaningful. But the best test of the benefits of improved dosimetric accuracy - an advantage in local control or survival - would require years of follow-up to observe.

\section{Conclusions}

Dosimetry calculations for IOHDR brachytherapy are typically carried out with treatment planning systems that assume an infinite scatter environment around the applicator and the target volume. We have shown this assumption results in significant shortening of the prescription depth, thereby leading to substantial underdosage to the tumor volume. The underdosage was found to be a function of the prescription depth and was found to be independent of the shape and area of the treated volume. It may be clinically relevant to correct for these errors by augmenting the scatter environment or, preferably, by appropriately modifying the prescription dose or by moving the dose prescription points downstream from the catheters during the treatment planning itself.

\section{References}

1. Palta JR, Biggs PJ, Hazle JD, Huq MS, Dahl RA, Ochran $\mathrm{TG}$, et al. Intraoperative electron beam radiation therapy: Technique, dosimetry, and dose specification: Report of Task Force 48 of the radiation therapy committee, American Association of Physicists in Medicine. Int J Radiat Oncol Biol Phys 1995; 33: 725-46.

2. Carvo FA, Micaily B, Brady LW. Intraoperative radiotherapy. A positive view. Am J Clin Oncol 1993; 16: 418-23.

3. Johnstone PA, Sindelar WF, Kinsella TJ. Experimental and clinical studies of intraoperative radiation therapy. Curr Probl Cancer 1994; 18: 249-90.

4. Willett CG. Intraoperative radiation therapy. Int J Clin Oncol 2001; 6: 209-14.

5. Konski A, Neisler J, Phibbs G, Bronn D, Dobelbower RR Jr.. The use of intraoperative electron beam radiation therapy in the treatment of para-aortic metastases from gynecologic tumors. Am J Clin Oncol 1993; 16: 67-71.

6. Goldson AL. Past, present, and future prospects of intraoperative radiotherapy (IORT). Semin Oncol 1981; 8: 59-64.

7. Toita T, Nakano M. Takizawa Y. Sueyama $H$, Kakihana Y, Kushi A, et al. Intraoperative radiation therapy (IORT) for head and Neck. Int J Radiat Oncol Biol Phys 1994; 30: 1219-24.

8. Dobelbower RR Jr., Konski AA, Merrick HW 3rd, Bronn DG, Schifeling D, Kamen C. Intraoperative electron beam radiation therapy (IOEBRT) for carcinoma of the exocrine pancreas. Int J Radiat Oncol Biol Phys 1991; 20: 113-19.

9. McCullough EC, Anderson JA. The dosimetric properties of an applicator system for intraoperative electron-beam therapy utilizing a Clinac-18 accelerator. Med Phys 1982; 9: 261-68.

10. Gieschen HL, Spiro IJ, Suit HD, Ott MJ, Rattner DW, Ancukiewicz M, et al. Long-term results of intraoperative electron beam radiotherapy for primary and recurrent retroperitoneal soft tissue sarcoma. Int J Radiat Oncol Biol Phys 2001; 50: 127-31. 
11. Nag S, Hu KS. Intraoperative high-dose-rate brachytherapy. Surg Oncol Clin N Am 2003; 12: 1079-97.

12. Nag S, Lukas P, Thomas DS, Harison L. Intraoperative high dose rate remote brachytherapy. In: Nag S, Editor. High dose rate brachytherapy: A textbook. Armonk, New York: Futura Publishing Company; 1994. p. 427-45.

13. Harrison LB, Minsky BD, Enker WE, Mychalczak B, Guillem J, Paty PB, et al. High dose rate intraoperative radiation therapy (HDR-IORT) as part of the management strategy for locally advanced primary and recurrent rectal cancer. Int J Radiat Oncol Biol Phys 1998; 42: 325-30.

14. Hu KS, Enker WE, Harrison LB. High-dose-rate intraoperative irradiation: current status and future directions. Semin Radiat Oncol 2002; 12: 62-80.

15. Nath R, Anderson LL, Luxton G, Weaver KA, Williamson JF, Meigooni AS. Dosimetry of interstitial brachytherapy sources: Recommendations of the AAPM Radiation Therapy Committee Task Group No. 43. Med Phys 1995; 22: 209-34.

16. Raina S, Avadhani JS, Oh M, Malhotra MK, Jaggernauth W, Kuettel MR, et al. Quantifying IOHDR brachytherapy underdosage resulting from an incomplete scatter environment. Int $J$ Radiat Oncol Biol Phys 2005; 61: 1582-86.

17. McDermott PN, He T, DeYoung A. Dose calculation accuracy of lung planning with a commercial IMRT treatment planning system. J Appl Clin Med Phys 2003; 4: 341-51.

18. Jacqueline E, Sasa M, William BH, Dempsey F, Low DA. Dosimetry of therapeutic photon beams using an extended dose range film. Med Phys 2002; 29: $2438-45$. 